\title{
FUZZY STRUCTURE OF COMPLEX BELIEF SYSTEMS: FUZZY RELATIONS AND FUZZY BELIEF SETS
}

Miguel Lloret-Climent, Sergio Pérez-Gonzaga, Josep-Lluis Usó-Doménech and JosuéAntonio Nescolarde-Selva

Department of Applied Mathematics, University of Alicante, Alicante, Spain

\begin{abstract}
In previous work (Nescolarde-Selva and Usó-Doménech, 2014 ${ }^{\mathrm{a}, \mathrm{b}}$ ) discussed the theory that complex belief systems have a topological structure. In this paper it is suggested that this structure is also fuzzy. They introduce the concepts of fuzzy sets in the context of beliefs (substantive and derived), and between derived beliefs themselves. Also introduced are the concepts of fuzzy covering, fuzzy invariance and the relationships between them.
\end{abstract}

Keywords: Belief systems, derived beliefs, fuzzy coverage, fuzzy relations, fuzzy invariability, ideology, substantive beliefs

\section{INTRODUCTION: PREVIOUS CONCEPTS}

Complex societies are concerned with possibility and necessity, their Superstructure can been divided in two as follows (Nescolarde-Selva and Usó-Doménech, 2013 ${ }^{\mathrm{a}, \mathrm{b}}, 2014^{\mathrm{a}, \mathrm{b}}$; Usó-Domènech and Nescolarde-Selva, 2012):

1) The Doxical Superstructure (DS) is formed by values in fact, political and religious ideologies and culture of a human society in a certain historical time.

2) The Mythical Superstructure(MS) also has been divided into two parts:

a) $\mathrm{MS}_{1}$ containing the mythical components or primigenial bases of the ideologies and cultures with the ideal values.

b) $\mathrm{MS}_{2}$ containing ideal values and utopias that are the wished and unattainable goals of belief systems of the Doxical Superstructure (DS).

These ideas are summarized in the following diagram (Figure 1): 


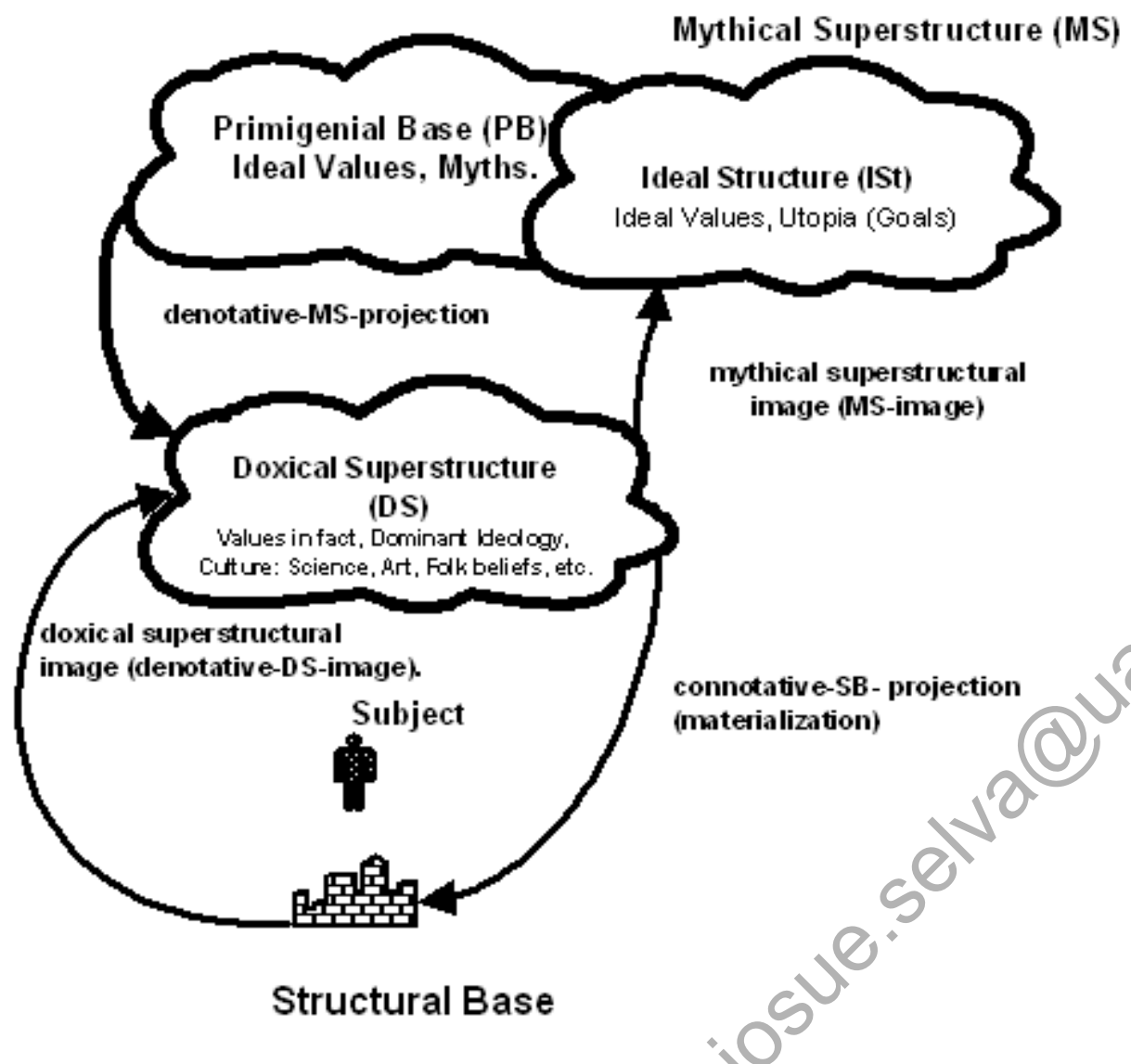

1: Structural base and superstructures.

Figure

According to Nescolarde-Selva and Usó-Doménech $\left(2013^{\mathrm{a}, \mathrm{b}}\right)$ a belief system is a set of related ideas, learned and shared which has some permanence in time and space, and to which individuals and/or groups exhibit some commitment (Borhek and Curtis, 1983; Eliade, 1978)). The conditions of permanence, commitment, and connectedness are variable characteristics through which we expect belief systems to be related to social organization.

Any belief system will be formed by two essential levels:

1. Ideal orabstract level.

2. Material level or text.

The first of the levels or the abstract level of the $S$ it is the focus of this work. It is embedded in the individual mind and has been acquired by means of physical transaction, either oral or visual, through a textual materialization, such as a written, pictorial, architectural, musical, and so forth, text. We may in fact affirm that a belief system operates as a cybernetic feedback process (Figure 2). 


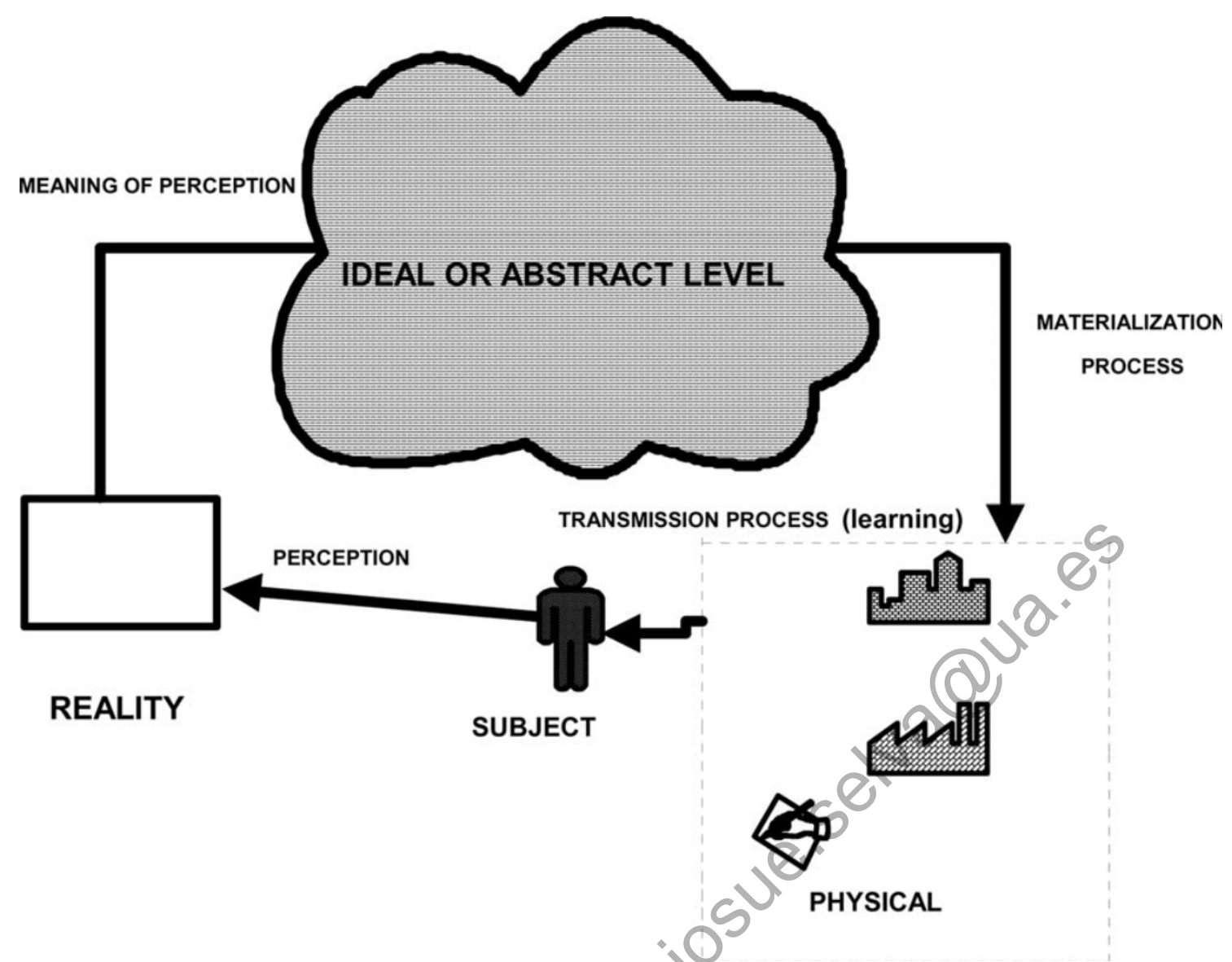

Figure 2: Cybernetic feedback process.

Definition 1: The abstract belief level) is formed by a set of elements nominated substantive beliefs $\Sigma$ forming the unquestionable truths of the system (axioms) and a set of derived beliefs $\Delta$, formed from substantive beliefs.

Substantive beliefs constitute the axioms of the system, while many of the derived beliefs will constitute their theorems.

Considering this argument we propose following initial hypotheses:

Hypothesis 1: Beliefs are not products of reason nor of abstract and logical thought.

Hypothesis 2: In the origin of any belief system there is always a supernatural system of beliefs.

Hypothesis 3: Derived beliefs become substantial beliefs with the passage of time, giving origin to a more or less ample body of substantive beliefs, that is to say, a religion.

Hypothesis 4: When belonging to the Ideological Doxical Superstructure (NescolardeSelva and Usó-Doménech.(2014 $\left.{ }^{a, b}\right)$ Usó-Doménech and Nescolarde-Selva, (2012), the set $S$ of substantive beliefs will be "ideal", that is to say, merely abstract.

Let $\Sigma=\left\{s_{1}, s_{2}, \ldots, s_{n}\right\}$ be the set of substantive beliefs and $\Delta=\left\{d_{1}, d_{2}, \ldots, d_{m}\right\}$ the set of derived beliefs, such that $B S=\Sigma \bigcup \Lambda=\left\{s_{1}, s_{2}, \ldots, s_{n}, d_{1}, d_{2}, \ldots, d_{m}\right\}$ (Nescolarde-Selva and 
Usó-Doménech, 2013). There is the "no belief" or empty belief, which we will represent by $\varnothing$. Set BS forms a belief sequence because it is an ordered list of objects. It contains terms or beliefs, and the number of terms is called the length of the sequence. Order matters, and the exact same terms can appear multiple times at different positions in the belief sequence. BS forms a finite sequence with terms in the set BS because it is a function from $\left\{s_{1}, s_{2}, \ldots, s_{n}, d_{1}, d_{2}, \ldots, d_{m}\right\}$ to BS.

Hypothesis 5: We consider the subset of substantive beliefs $\Sigma$ as fixed, i.e., with a constant cardinal, and the subset of derived beliefs $D$ possessing a varying cardinal.

We shall say that a set of variables $\left\{x_{1}, \ldots, x_{n}\right\}$ forms a chain of relations if there is a set of relations $\left\{r_{1}, \ldots, r_{n-1}\right\} \in R$ such that $\left\{x_{1} r_{1} x_{2}, x_{2} r_{2} x_{3}, \ldots, x_{n-1} r_{n-1} x_{n}\right\}$. The belief system thus conceived, will present a chain structure (Figure 3 ).
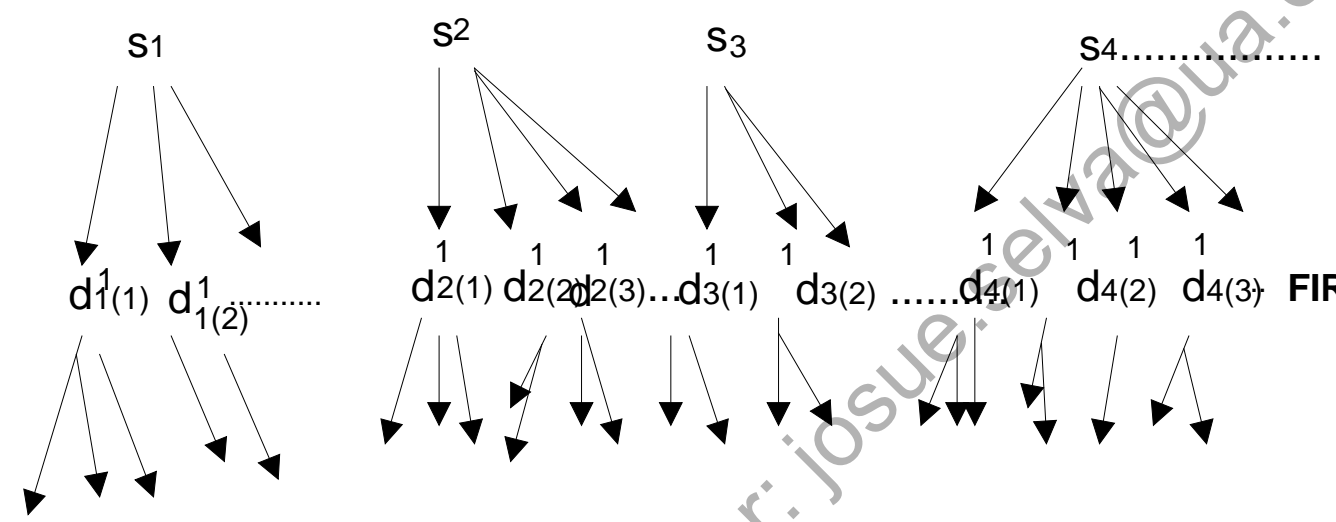

$d_{4(1)} \quad d_{4}(2) \quad d 4(3)$ FIRST LEVEL

SECOND LEVEL

THIRD LEVEL

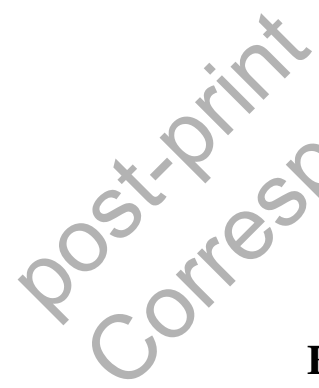

Figure 3: Chain structure of belief systems.

Therefore it will constitute an alysidal set as defined by Nescolarde-Selva, Vives Maciá, Usó-Doménech and Berend, (2012 $\left.{ }^{\mathrm{a}, \mathrm{b}}\right)$ and Nescolarde-Selva and Usó-Doménech (2012)

Definition 1: A belief system is an alysidal belief set (S,R) whose elements are chains formed by beliefs united by freeways of relations of logical derivation.

Note 1: The sheaf is a single relation (monorelational sheaf).

Note 2: It is a clockwise sheaf (sheaf of direct relations) denoted $d$ - $h_{i j}$ because relations forming the sheaf go from element $x_{i}$ to element $x_{j}$. 
Due to the complexity of the proposed problem, we have taken a decision to simplify the chains, assuming that each substantive belief s, produces a single chain of derived beliefs (Figure 4).

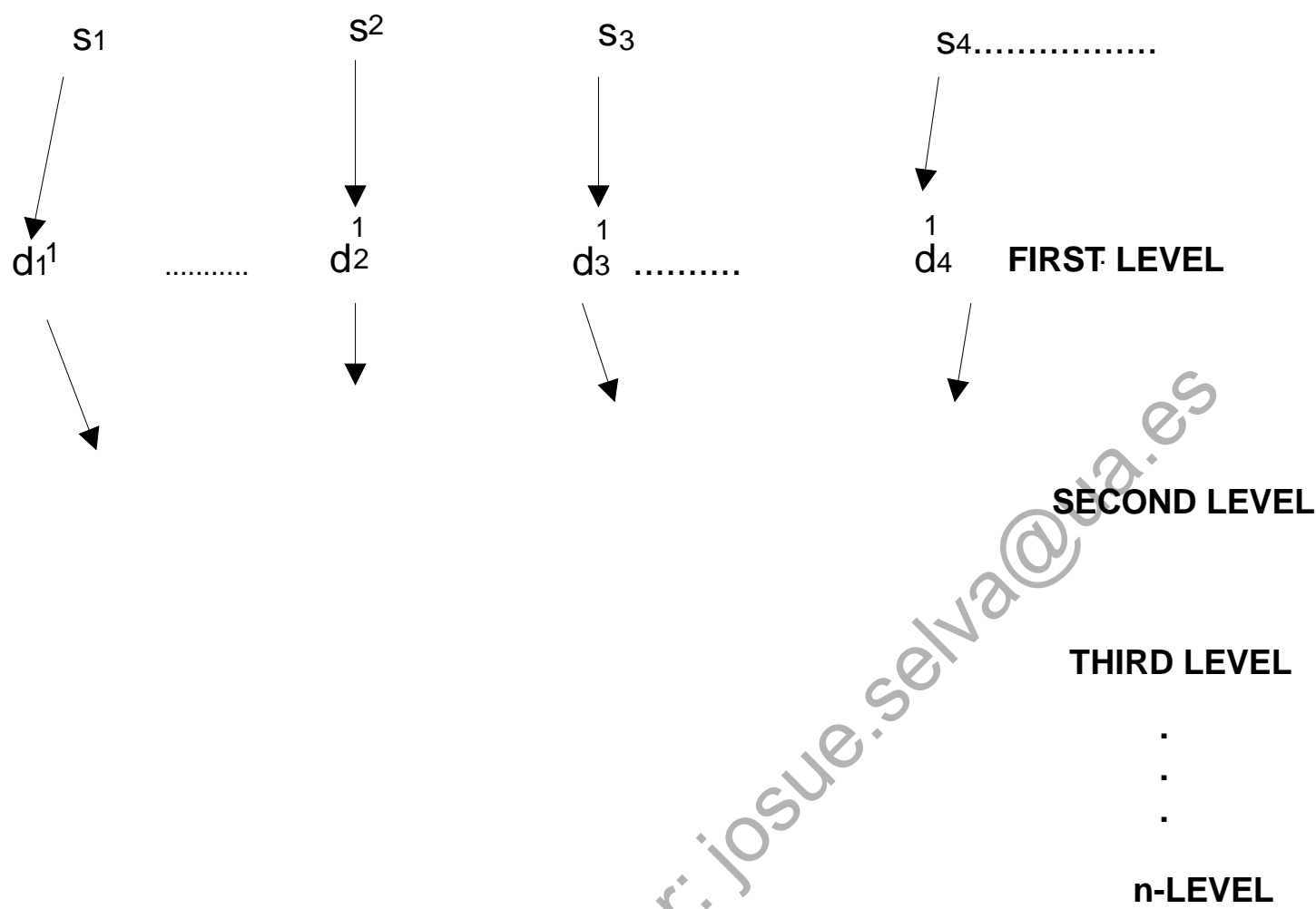

Figure 4: Simplified structure of chain of belief systems.

\section{FUZZY DERIVED RELATIONS}

A fuzzy relation is characterized by the same two items in a fuzzy set:

1) It is a list containing element and membership grade pairs, $\left\{\left\{\mathrm{v}_{1}, w_{1}\right\}, \mathrm{R}_{11}\right\},\left\{\left\{\mathrm{v}_{1}, \mathrm{w}_{2}\right.\right.$ \},$\left.\left.R_{12}\right\}, \ldots,\left\{\left\{v_{m}, w_{m}\right\}, R_{r_{m}}\right\}\right\}$. Note that the elements of the relation are defined as ordered pairs, $\left\{\mathrm{v}_{1}, \mathrm{w}_{1}\right\},\left\{\mathrm{v}_{1}, \mathrm{w}_{2}\right\}, \ldots,\left\{\mathrm{v}_{\mathrm{n}}, \mathrm{w}_{\mathrm{m}}\right\}$. These elements are again grouped with their membership grades, $\left\{R_{11}, R_{12}, \ldots, R_{\text {rm }}\right\}$, which are values that range from 0 to 1 , inclusive.

2) The second item characterizing fuzzy relations is the universal space. For relations, the universal space consists of a pair of ordered pairs, $\left\{\left\{\mathrm{V}_{\min }, V_{\max }, c_{1}\right\},\left\{\mathrm{W}_{\min }, \mathrm{W}_{\max }, c_{2}\right\}\right\}$. The first pair defines the universal space to be used for the first set under consideration in the relation, and the second pair defines the universal space for the second set.

Assuming that $\mathrm{V}$ and $\mathrm{W}$ are two collections of objects, an arbitrary fuzzy set $\mathrm{B}$, defined as the Cartesian product VxW, will be called a fuzzy relation in the space VxW. $\mathrm{R}$ is thus a function defined in the space $V x W$, which takes values from the interval $[0,1]$.

$\mathrm{R}: \mathrm{V} \times \mathrm{W} \rightarrow[0,1]$. In the case where $\mathrm{V}=\mathrm{W}$, we have a binary fuzzy relation on a single set $\mathrm{V}$. We can start our discussion by considering a countable collection of objects $V=\left\{v_{i}\right\}, i=1,2, \ldots$ Then $W=\left\{w_{j}\right\}, j=1,2, \ldots$ 
A fuzzy relation $R$ can be represented in the following way: $R=\left\{\left\{\left\{\mathrm{v}_{i}, w_{j}\right\}, R\left(v_{i}, w_{j}\right)\right\}\right\}$, $\mathrm{i}=1,2, \ldots ; \mathrm{j}=1,2, \ldots$

According to Lloret-Climent, Nescolarde-Selva and Pérez-Gonzaga (2014), the direct and indirect influences between beliefs will provide the basis for obtaining fuzzy and/or non-fuzzy relationships, so that the concepts of coverage and invariability between sets of beliefs will appear naturally, these concepts and their interconnections will be analyzed from the viewpoint of algebraic properties of inclusion, union and intersection, as well as with the loop concept, which as we shall see, will be especially important. In our work, we use some important concepts of the fuzzy system, including membership function, inclusion, intersection, union, support and fuzzy relation.

When any two beliefs are related, we know that one of them exercises, directly, a certain influence on the other. Taking this into account, we obtain the following definitions and results, bearing in mind the fact that the relations will always be fuzzy from now on since we can always consider a classic relation as a particular case of a fuzzy one.

Let $B=(S, R)$ be a belief system. Let $s_{i}(i=1,2, \ldots n)$ be the substantive beliefs and $d_{i}^{k}$ the derived beliefs. The subscript $\mathrm{i}=1,2, \ldots, \mathrm{n}$ corresponds to the substantive belief which derives and the superscript $\mathrm{k}=1,2, \ldots, \mathrm{m}$ indicates the level of derived belief.

Definition 2: If two beliefs $s_{i}, d_{i}^{1} \in S$ and $d_{i}^{k}, d_{i}^{k+1} \in S$ verify that $\exists r_{i} \in R 7 \mu_{r}\left(s_{i}, d_{i}^{1}\right)=p>0$ or $\mu_{r}\left(d_{i}^{k}, d_{i}^{k+1}\right)=p>0$ for any fuzzy relation, we say that $s_{i}$ directly influences $d_{i}^{1}$ or $d_{j}^{k}$ directly influences $d_{j}^{k+1}$ with grade $p$ through the fuzzy relation $r$.

From now on, we need to bear in mind the fact that the chains of relations that will exist between any two beliefs of $\mathrm{S}$ may contain classic or fuzzy relations of the types mentioned here in indiscriminate fashion, as well as containing elements that could belong to fuzzy belief sets or not. Clearly, all the degrees of influence we are going to consider will strictly be greater than zero. If not, we would have superfluous influences, so we eliminate them and simplify the chain of relations.

Definition 3: A belief $s_{i} \in S$ indirectly influences the element $d_{i}^{k} \in S$ with grade $p$, if there is a chain, starting with $s_{1}$ and ending with $d_{i}{ }^{2}$, that contains relations of $R$ and elements of $S$, and verifying that the minimum degree of indirect influence obtained with this chain is equal to $p$.

Definition 4: The structure of the belief system is hierarchical because no subset of $S$ is a loop.

Definition 5: The structure of relations for each derived belief is:

$$
f_{S}\left(s_{i}\right)=\left\{d_{i}^{1} \in S / \exists r_{i}^{1} \in R, \mu_{r}\left(s_{i}, d_{i}^{1}\right)>0\right\} \wedge f_{S}\left(d_{i}^{k}\right)=\left\{d_{i}^{k+1} \in S / \exists r_{i}^{k} \in R, \mu_{r}\left(d_{i}^{k}, d_{i}^{k+1}\right)>0\right\}
$$

Definition 6: The structure of relations for the derived belief $d_{i}^{1} \in S$ associated with the fuzzy relation $r_{i}^{k} \in R$ is $f_{S}\left(d_{i}^{k}, r_{i}^{k}\right)=\left\{d_{i}^{k+1} \in S / \mu_{r}\left(d_{i}^{k}, d_{i}^{k+1}\right)>0\right\}$. 
Definition 7: The structure of relations for any belief set $B \subseteq S$ is $f_{S}(B)=\bigcup_{\substack{s_{i} \in B \\ d_{i}^{k} \in B}} f_{S}\left(s_{i}\right) f_{S}\left(d_{i}^{k}\right)$.

Definition 8: The structure of relations for any set $B \subseteq S$ and associated with the fuzzy relation $r_{i}^{k} \in R$ is $f_{S}\left(B, r_{i}^{k}\right)=\bigcup_{\substack{s_{i} \in B \\ d_{i}^{k} \in B}} f_{S}\left(s_{i}, r_{i}^{1}\right) f_{S}\left(d_{i}^{k}, r_{i}^{k}\right)$.

From the above we can deduce the following result:

Theorem 1: For each $B \subseteq S$ is verified that $f_{S}(B)=\bigcup_{r_{i}^{k} \in R} f_{S}\left(B, r_{i}^{k}\right)$.

Proof.

$$
\bigcup_{r_{i}^{k} \in R} f_{S}\left(B, r_{i}^{k}\right)=\bigcup_{r_{i}^{k} \in R}\left(\bigcup_{\substack{s_{i} \in B \\ d_{i}^{k} \in B}} f_{S}\left(s_{i}, r_{i}^{1}\right) f_{S}\left(d_{i}^{k}, r_{i}^{k}\right)\right)=\bigcup_{r_{i}^{k} \in B} f_{S}\left(s_{i}\right) f_{S}\left(d_{i}^{k}\right)=f_{S}(B) .
$$

\section{FUZZY DERIVED BELIEFS' SETS}

Fuzzy set theory shows the intention to generalizethe classical notion of a set and a proposition to accommodate fuzziness in the sense that it is contained in human language, that is, in human beliefs, judgments, evaluations, and decisions. Zadeh writes (1965): "The notion of a fuzzy set provides a convenient point of departure for the construction of a conceptual framework which parallels in many respects the framework used in the case of ordinary sets, but it is more general than the latter and, potentially, may prove to have a much wider scope of applicability, particularly in the fields of pattern classification and information processing. Essentially, such a framework provides a natural way of dealing with problems in which the source of imprecision is the absence of sharply defined criteria of class membership rather than the presence of random variables".

Most of us are familiar with Classical logic. Classical logic is bivalued. That is, propositions are seen as either True or False. So, when we reason about propositions like "Sarah is a woman." We can evaluate that proposition as either True or False. But fuzzy logic is many-valued. Many-valued logic (Béziau, 1997; Cignoli et al, 2000; Malinowski, 2001; Miller and Thornton, 2008; Usó-Doménech, Nescolarde-Selva and Pérez-Gonzaga, 2014) differs from classical logic from the fundamental fact that it allows for partial truth. In classical logic, truth takes on values in the set $\{0,1\}$, in other words, only the value 1 or 0 , meaning "Yes, it's true," or "No, it's not," respectively. It is a t-norm based system. Many-valued logics as their natural extension which take on values in the interval $[0,1]$. Per definition, t-norm based systems are many-valued if the set of valuations is not countable and this set is the interval $[0,1]$. That is, setmembership can vary along a continuum. "Truthfulness" can vary along a continuum from $100 \%$ True to $50 \%$ True to $0 \%$ True (and all values in between). This may seem strange, but examples are close at hand. For example, evaluate the following propositions. Are they true or false? "Dani is a good person." "This conversation is gossip." "Isaac is a Jewish." "That view is heresy." "Sarah believes in God." Are 
propositions like these, moral and ideological propositions, best evaluated by classical, bivalued logic? Can we cleanly say that a given proposition is true or false? The reason is that sets such as "goodness," "gossip," "Jewish," "heresy," and "belief" are best viewed as fuzzy sets. Fuzzy sets don't have discrete boundaries. Thus, set-membership is not a discrete In versus Out. Extreme cases might be easily classified, but generally we would evaluate set-membership as varying along a continuum.

Example 1: "Teacher, which is the greatest commandment in the Law?" Jesus replied: "'Love the Lord your God with all your heart and with all your soul and with all your mind.' This is the first and greatest commandment. And the second is like it: 'Love your neighbor as yourself.' All the Law and the Prophets hang on these two commandments." (Matthew 22: 36-40)

Lots of doctrinal and ethical propositions are true. But we like the example above because they hint that doctrinal and ethical categories are fuzzy. That is, there are "greatest" commandments and things of "first" importance. Too often, in doctrinal and ethical discussions we get tangled in, people tend to reason and debate in bivalued categories. Of course, if a proposition is in the Bible it can lay a claim to truth. But these passages, and the example we offered above, suggest that debates and conversations may be better served if people employ fuzzy categories. Allowing some issues to be, we have no better words for this, "more true" or "more ethical"

Dominance of bivalued logic is why the public discourse involving ideology is so impoverished. Bivalued logic is neat, clean, and easy to use. Everything is cut and dried. There is good and there is evil. And whose sideare you on? There is right and there is wrong. Which will you choose? Etc.

The ability to rationally change one's beliefs in the face of new information which, possibly, contradicts the currently held beliefs is a basic characteristic of intelligent behavior. A very successful framework in which this question is studied is the one due to Alchourrón, Gärdenfors and Makinson (1985) and Gärdenfors (1988), with its operation of partial meet revision. One limitation of this framework is that belief in a formula is taken as a matter of all or nothing: either the formula is believed or it is not. One limitation of this framework is that belief in a formula is taken as a matter of all or nothing: either the formula is believed or it is not. However, real-life knowledge bases may well contain information of a more graded nature. For instance we might want to represent information about vague concepts or uncertain beliefs. Likewise revision inputs may come with a degree of certainty attached.

Definition 9: If $S$ is a collection of derived beliefs denoted generically by $d_{i}^{k}$, then $a$ fuzzy belief set $\bar{A}$ in $S$ is a set of ordered pairs: $\bar{A}=\left\{\left(d_{i}^{k}, \mu_{\bar{A}}\left(d_{i}^{k}\right) \mid d_{i}^{k} \in S\right)\right\}$

$\mu_{\bar{A}}\left(d_{i}^{k}\right)$ is called the membership function (generalized characteristic function) which maps $\mathrm{S}$ to the membership space $\mathrm{M}$. Its range is the subset of non-negative real numbers whose supremum is finite. For sup $\mu_{\bar{A}}\left(d_{i}^{k}\right)=1$ : normalized fuzzy set.

In this definition, the membership function of the fuzzy set is a real-valued function. A type $m$ fuzzy belief set whose memberships value are type $m-1, m>1$, fuzzy belief sets on $[0,1]$. 


\section{FUZZY COVERAGE}

One of the first properties to appear when studying systemic approachs of complex systems is coverage (Esteve and Lloret, 2006 ${ }^{\mathrm{a}, \mathrm{b}}$ ). This property is fundamental when studying the direct relations existing between all the subsets of $S$.

Definition 10: Let $A, B \subseteq S$ be two sets of beliefs. We say that $A$ covers $B$ if $f_{S}(A)=B$. In other words, if each belief element of $B$ is directly influenced by any belief element of $A$.

For the case where the sets $A$ and $B$ are fuzzy $(\bar{A}, \bar{B})$, the above definition becomes $f_{s}$ $(\sup p(\bar{A}))=\sup p(\bar{B})$, as for any belief $s_{i}, d_{i}^{k} \in \bar{A}$ whose membership function-is 0 , we obtain $\mu_{r}\left(s_{i}, d_{j}^{k}\right) \leq \min \left(\mu_{\bar{A}}\left(s_{i}\right), \mu_{\bar{B}}\left(d_{j}^{k}\right)\right)=\min \left(0, \mu_{\bar{B}}\left(d_{j}^{k}\right)\right)$ or $\mu_{r}\left(d_{i}^{k}, d_{j}^{l}\right) \leq \min \left(\mu_{\bar{A}}\left(d_{i}^{k}\right), \mu_{\bar{B}}\left(d_{j}^{l}\right)\right)=\min \left(0, \mu_{\bar{B}}\left(d_{j}^{l}\right)\right)$, so that $\mu_{r}\left(s_{i}, d_{j}^{k}\right)=0$, and $s_{i}$ will not be effectively related to any other belief or $\mu_{r}\left(d_{i}^{k}, d_{j}^{l}\right)=0$ and $d^{k}$ will not be effectively related to any other belief.

However, if set $\bar{A}$ is fuzzy and $B$ is non-fuzzy, the definition of coverage becomes: $f_{S}(\sup p(\bar{A}))=B$.

Superscripts $k$, l can be $k=l$ or $k \neq l$.

Theorem 2: Let $\bar{A}, \bar{B}, \bar{C}$ be the fuzzy beliefs sets such as $\bar{A}, \bar{B}, \bar{C} \subseteq S$. If $\bar{A}$ covers $\bar{B}$ and $\bar{B}$ covers $\bar{C}$, then each element of sup $p(\bar{C})$ is indirectly influenced by an element of $\sup p(\bar{A})$.

Proof.

Let $d_{h}^{m} \in \sup p(\bar{C})$. As $\bar{B}$ covers $\bar{C}$, we obtain $\exists d_{j}^{l} \in \sup p(\bar{B}) \wedge \exists r \in R$, so that $\mu_{r}\left(d_{j}^{l}, d_{h}^{m}\right)>0$. Similarly, as $d_{j}^{l} \in \sup p(\bar{B})$ and $\bar{A}$ covers $\bar{B}$, we obtain $\exists d_{i}^{k} \in \sup p(\overline{\bar{A}}) \wedge \exists r \in R$, so that $\mu_{r}\left(d_{i}^{k}, d_{j}^{l}\right)>0$.As $\mu_{r}\left(d_{i}^{k}, d_{j}^{l}\right)>0 \wedge \mu_{r}\left(d_{j}^{l}, d_{h}^{m}\right)$, we therefore deduce that $d_{i}^{k}$ exercises an indirect influence on $d_{h}^{m}$. Reasoning in the same way about each element of sup $p(\bar{C})$ demonstrates the theorem.

Note 1: The result we have just proven is also valid for combinations of the three belief sets $A, B$ and $C$ that are not necessarily fuzzy beliefs sets, for example taking $\bar{A}$ and $\bar{C}$ as fuzzy belief sets and $B$ as a non-fuzzy belief set.

Using the property of coverage, the possible existence of loops is determined in the following result:

Theorem 3: Let $\bar{A}, \bar{B} \subseteq S$ be the belief fuzzy sets, where sup $p(\bar{A})$ is a finite set, so that sup $p(\bar{A}) \subseteq \sup p(\bar{B})$. Thus, if $\bar{A}$ covers $\bar{B}$, a loop will be included in sup $p(\bar{A})$.

Proof. 
We know that $\sup p(\bar{A}) \subseteq \sup p(\bar{B})=f_{S}(\sup p(\bar{A}))$, as $\bar{A}$ covers $\bar{B}$.

Let $n=\operatorname{card}(\sup p(\bar{A}))$, and let $d_{n+1}$ be any derived belief of sup $p(\bar{A})$. Thus, $d_{n+1} \in f_{S}(\sup p(\bar{A}))$, so that $\exists d_{n+1} \in \sup p(\bar{A})$ and $\exists r_{n} \in R$, so that $\mu_{r}\left(d_{n}, d_{n+1}\right)>0$. In addition, as $d_{n} \in f_{S}(\sup p(\bar{A})), \exists d_{n+1} \in \sup p(\bar{A})$ and $\exists d_{n-1} \in R \exists r_{n-1} \in R$, so that $\mu_{r}\left(d_{n-1}, d_{n}\right)>0$.

After $n$ iterations, we obtain a chain of relations and, as the cardinal of $\sup p(\bar{A})$ is $n$, we obtain $\exists i, j \in\{1,2, \ldots, n+1, i<j\}$, so that $d_{i}=d_{j}$ and therefore the set $d_{i}, \ldots, d_{j}$ forms a loop contained in $\sup p(\bar{A})$.

Note 2: The above property is also valid if one of the beliefs sets is fuzzy and the other is not.

Theorem 4: Let $\bar{A}, \bar{B} \subseteq S$ be two fuzzy belief sets, verifying that $\bar{A} \subseteq \bar{B}$. Thus, $\sup p(\bar{A}) \subset \sup p(\bar{B})$.

Proof.

As $\bar{A} \subseteq \bar{B}$, then $\mu_{\bar{A}}\left(d_{i}^{k}\right) \leq \mu_{\bar{B}}\left(d_{i}^{k}\right) \forall d_{i}^{k} \in S$. In addition, if $d_{i}^{k} \in \sup p(\bar{A})$, we know that $\mu_{\bar{A}}\left(d_{i}^{k}\right)>0$ and thus, $\mu_{\bar{B}}\left(d_{i}^{k}\right) \geq \mu_{\bar{A}}\left(d_{i}^{k}\right)>0$, so obtaining $d_{i}^{k} \in \sup p(\bar{B})$.

\section{FUZZY INVARIABILITY}

In previous articles (Esteve and Lloret, 2006 ${ }^{\mathrm{a}, \mathrm{b}}$ ) have shown that the abundance of invariable subsets of $S$ can guarantee system behavior with little predisposition to change, and therefore free of upsets. We will now adapt the definition of the invariable belief subset to our fuzzy environment.

Definition 11: Let $\bar{A} \subseteq S$ be a fuzzy belief set. We say that sup $p(\bar{A})$ is invariable if $f_{S}(\sup p(\bar{A})) \Subset \sup p(\bar{A})$.

Note 3: If $A$ is and ordinary belief set, $A$ is invariable if $f_{S}(A) \subseteq A$.

Theorem 5: Let $\bar{A} \subseteq S$ be a fuzzy belief set such that sup $p(\bar{A})$ is invariable. Thus, $f_{S}(\sup p(\bar{A}))$ is also invariable.

\section{Proof}

Let $d_{i}^{k} \in f_{S}\left(f_{S}(\sup p(\bar{A}))\right.$. Thus $\exists d_{i}^{l} \in f_{S}\left(\sup p(\bar{A}) / d_{i}^{k} \in f_{S}\left(d_{i}^{l}\right)\right.$. As sup $p(\bar{A})$ is invariable, we obtain $d_{i}^{l} \in \sup p(\bar{A})$, and also $d_{i}^{k} \in f_{S}$ (sup $p(\bar{A})$ ), which proves that $f_{S}(\sup p(\bar{A}))$ is invariable. 
Note 4: The above theorem can also be extended to $f_{S}\left(f_{S}(\sup p(\bar{A}))\right)$, and in this way indefinitely to $\left.f_{S}\left(f_{S}\left(\ldots\left(\ldots f_{S}(\sup p(\bar{A}))\right) \ldots\right) \ldots\right)\right)$

Theorem 6: Let $\bar{A}, \bar{B} \subseteq S$ be two fuzzy belief sets, whose supports are both invariable. Thus, sup $p(\bar{A}) \cup \sup p(\bar{B})$ for the ordinary union is invariable.

Proof

Let $d_{i}^{k} \in \sup p(\bar{A}) \bigcup \sup p(\bar{B})$, thus $d_{i}^{k} \in \sup p(\bar{A}) \vee d_{i}^{k} \in \sup p(\bar{B})$. If $d_{i}^{k} \in \sup p(\bar{A})$, as sup $p(\bar{A})$ is invariable, we obtain $f_{S}(\sup p(\bar{A})) \subseteq \sup p(\bar{A})$, and thus $f_{s}\left(d_{i}^{k}\right) \subseteq \sup p(\bar{A}) \cup \sup p(\bar{B})$. For $d_{i}^{k} \in \sup p(\bar{B})$, the same reasoning is applied and we complete the demonstration.

Theorem 7: The fuzzy and ordinary unions and the support satisfy sup $p(\bar{A}) \cup \sup p(\bar{B})=\sup p(\bar{A} \cup \bar{B})$.

Proof

As

$d_{i}^{k} \in \sup p(\bar{A}) \bigcup \sup p(\bar{B}) \Leftrightarrow d_{i}^{k} \in \sup p(\bar{A}) \vee d_{i}^{k} \in \sup p(\bar{B}) \Leftrightarrow \mu_{\bar{A}}\left(d_{i}^{k}\right)>0 \vee \mu_{\bar{B}}\left(d_{i}^{k}\right)>0 \Leftrightarrow$ $\max \left(\mu_{\bar{A}}\left(d_{i}^{k}\right), \mu_{\bar{B}}\left(d_{i}^{k}\right)\right)>0 \Leftrightarrow d_{i}^{k} \in \sup p(\bar{A} \cup \bar{B})$

Theorem 8: Let $\bar{A}, \bar{B} \subseteq S$ be two fuzzy belief sets both with invariable supports. Thus, sup $p(\bar{A} \cup \bar{B})$ is invariable.

Proof

It is deduced from the twoprevious theorems.

The same result is obtained with the intersection of two invariable sets and we will now show this.

Theorem 9: Let $\bar{A}, \bar{B} \subseteq S$ be two fuzzy belief sets both with invariable supports. Thus, ifsup $p(\bar{A}) \bigcap \sup p(\bar{B}) \neq \varnothing$, we see that sup $p(\bar{A}) \bigcap \sup p(\bar{B})$ is invariable for the ordinary intersection.

Proof

Let $d_{i}^{k} \in \sup p(\bar{A}) \bigcap \sup p(\bar{B})$. As $d_{i}^{k} \in \sup p(\bar{A})$ and sup $p(\bar{A})$ is invariable, $f_{S}\left(d_{i}^{k}\right) \subseteq \sup p(\bar{A})$. In the same way, with $d_{i}^{k} \in \sup p(\bar{B})$ we obtain $f_{s}\left(d_{i}^{k}\right) \subseteq \sup p(\bar{B})$. 
Thus $f_{S}\left(d_{i}^{k}\right) \subseteq \sup p(\bar{A}) \bigcap \sup p(\bar{B})$,

giving $f_{s}(\sup p(\bar{A}) \bigcap \sup p(\bar{B})) \subseteq \sup p(\bar{A}) \bigcap \sup p(\bar{B})$, and thus sup $p(\bar{A}) \bigcap \sup p(\bar{B})$

will be invariable.

Theorem 10: The ordinary and fuzzy intersections and the support satisfy $\sup p(\bar{A}) \cap \sup p(\bar{B})=\sup p(\bar{A} \cap \bar{B})$.

Proof

As

$d_{i}^{k} \in \sup p(\bar{A}) \bigcap \sup p(\bar{B}) \Leftrightarrow d_{i}^{k} \in \sup p(\bar{A}) \wedge d_{i}^{k} \in \sup p(\bar{B}) \Leftrightarrow \mu_{\bar{A}}\left(d_{i}^{k}\right)>0 \wedge \mu_{\bar{B}}\left(d_{i}^{k}\right)>0 \Leftrightarrow$ $\min \left(\mu_{\bar{A}}\left(d_{i}^{k}\right), \mu_{\bar{B}}\left(d_{i}^{k}\right)\right)>0 \Leftrightarrow d_{i}^{k} \in \sup p(\bar{A} \cap \bar{B})$.

Theorem 11: Let $\bar{A}, \bar{B} \subseteq S$ be two fuzzy belief sets, whose supports are both invariable. Thus, if sup $p(\bar{A}) \cap \sup p(\bar{B}) \neq \varnothing$, sup $p(\bar{A} \cap \bar{B})$ is invariable.

Proof

It is deduced from the last two theorems.

\section{RELATIONS BETWEEN FUZZY CCOVERAGE AND FUZZY INVARIABILITY IN FUZZY DERIVED BELIEF SETS}

We now present a series of results, from different points of view, which prove the relation existing between the concepts of coverage and invariability in fuzzy derived belief sets.

Let (S, R) be a belief system and Met $\bar{A}, \bar{B} \subseteq S$ be two fuzzy belief sets both with invariable supports

Theorem 12: If $\bar{A}$ covers $\bar{B}$, sup $p(\bar{A}) \bigcup \sup p(\bar{B})$ will be invariable.

Proof

Let $d_{i}^{k} \in \sup p(\bar{A}) \bigcup \sup p(\bar{B})$, thus $d_{i}^{k} \in \sup p(\bar{A}) \vee d_{i}^{k} \in \sup p(\bar{B})$. If $d_{i}^{k} \in \sup p(\bar{A})$, as $\bar{A}$ covers $\bar{B}$, then $f_{s}\left(d_{i}^{k}\right) \subseteq \sup p(\bar{A}) \subseteq \sup p(\bar{A}) \cup \sup p(\bar{B})$. In addition, if $d_{i}^{k} \in \sup p(\bar{B})$, as this is an invariable set, we get $f_{S}\left(d_{i}^{k}\right) \subseteq \sup p(\bar{B}) \subseteq \sup p(\bar{A}) \cup \sup p(\bar{B})$. All this proves that sup $p(\bar{A}) \cup \sup p(\bar{B})$ is invariable.

Corollary 1: Under the same conditions as the previous Theorem, we have sup $p(\bar{A}) \bigcup \sup p(\bar{B})$ covering sup $p(\bar{B})$.

Proof

Trivial, applying properties. 
Let $\bar{A}, \bar{B} \subseteq S$ be two fuzzy belief sets so that $\bar{A}$ covers $\bar{B}$.

Theorem 13: $\operatorname{Supp}(\bar{A})$ will be invariable $\Leftrightarrow \sup p(\bar{B}) \subseteq \sup p(\bar{A})$.

Proof

$(\Rightarrow)$ Let $d_{i}^{k} \in \operatorname{Supp}(\bar{A})$. As $\bar{A}$ covers $\bar{B}$ we obtain $\exists d_{i}^{l} \in \operatorname{Supp}(\bar{A})$, so that

$d_{i}^{k} \in f_{S}\left(d_{i}^{l}\right)$. In addition, as sup $p(\bar{A})$ is invariable, $d_{i}^{k} \in f_{S}\left(d_{i}^{l}\right) \subseteq \sup p(\bar{A})$. In other words, $\Leftrightarrow \sup p(\bar{B}) \subseteq \sup p(\bar{A})$.

$(\Leftarrow)$ Let $d_{i}^{k} \in \operatorname{Supp}(\bar{A})$. As $\bar{A}$ covers $\bar{B}$ we obtain $f_{s}\left(d_{i}^{k}\right) \subseteq \sup p(\bar{B}) \subseteq \sup p(\bar{A})$. Thus, $f_{S}(\sup p(\bar{B}) \subseteq \sup p(\bar{A})) \wedge \sup p(\bar{A})$ will be invariable.

Let $\bar{A} \subseteq S$ be the fuzzy belief set, so that $\bar{A}$ covers itself.

Corollary 2: $\operatorname{Supp}(\bar{A})$ is invariable.

Proof

Trivial, replacing $\bar{A}$ with $\bar{A}$ in the previous theorem.

Let $\bar{A}, \bar{B} \subseteq S$ be two fuzzy belief sets so that $\vec{A}$ covers $\bar{B}$ and $\bar{B}$ covers $\bar{A}$

Theorem 14: $\operatorname{Supp}(\bar{A}) \bigcup \sup p(\bar{B})$ is invariable.

Proof

Let $d_{i}^{k} \in \sup p(\bar{A}) \cup \sup p(\bar{B})$, thus $d_{i}^{k} \in \sup p(\bar{A}) \vee d_{i}^{k} \in \sup p(\bar{B})$. If $d_{i}^{k} \in \sup p(\bar{A})$, we obtain $f_{S}\left(d_{i}^{k}\right) \subseteq \sup p(\bar{B}) \subseteq \sup p(\bar{A}) \cup \sup p(\bar{B})$. In addition, if $d_{i}^{k} \in \sup p(\bar{B})$, we obtain $f_{s}\left(d_{i}^{k}\right) \subseteq \sup p(\bar{A}) \subseteq \sup p(\bar{A}) \cup \sup p(\bar{B})$.

Thus, $\forall d_{i}^{k} \in \sup p(\bar{A}) \bigcup \sup p(\bar{B})$ verifies that $f_{S}\left(d_{i}^{k}\right) \subseteq \sup p(\bar{A}) \bigcup \sup p(\bar{B})$, giving $f_{S}(\sup p(\bar{A}) \cup \sup p(\bar{B})) \subseteq \sup p(\bar{A}) \bigcup \sup p(\bar{B})$, and therefore sup $p(\bar{A}) \cup \sup p(\bar{B})$ will be invariable.

We obtain a similar but more enlarged result with the following corollary.

Corollary 3: Let $\bar{A}_{o}, \bar{A}_{1}, \bar{A}_{2}, \ldots, \bar{A}_{n} \subseteq S$ be the fuzzy belief sets, so that $\bar{A}_{o}=\bar{A}_{n}$ and $\bar{A}_{i}$ cover $\bar{A}_{i+1}, \quad \forall i=0,1, \ldots, n-1$. Thus, sup $p\left(\bar{A}_{0}\right) \bigcup \sup p\left(\bar{A}_{1}\right) \cup \ldots \cup \sup p\left(\bar{A}_{n-1}\right)$ is invariable.

Proof 
Trivial, applying the known properties and using the same reasoning as in the previous Theorem.

Theorem 15: Let $\bar{A}, \bar{L} \subseteq S$ be fuzzy belief sets, so that sup $p(\bar{A})$ is invariable and sup $p(\bar{L})$ is a loop. Thus, it can only be that sup $p(\bar{L}) \subseteq \sup p(\bar{A})$ or that $\sup p(\bar{A})$ and sup $p(\bar{L})$ are disjointed.

Proof

Let $d_{i}^{k} \in \sup p(\bar{L})$. We can find that $d_{i}^{k} \in \sup p(\bar{A}) \vee d_{i}^{k} \notin \sup p(\bar{A})$. Firstly, suppose that $d_{i}^{k} \in \sup p(\bar{A})$. As sup $p(\bar{A})$ is invariable, we will have $f_{S}\left(d_{i}^{k}\right) \subseteq \sup p(\bar{A})$. In other words, the derived belief following $d_{i}^{k}$ in the loop will also belong to $\sup p(\bar{A})$. Using the same reasoning, the following derived belief will also belong to sup $p(\bar{A})$ and successively until we reach our derived belief $d_{i}^{k}$. All derived beliefs in the loop therefore belong to sup $p(\bar{A})$ and we obtain that sup $p(\bar{L}) \subseteq \sup p(\bar{A})$. Now suppose that $d_{i}^{k} \notin \sup p(\bar{A})$. In this case, if the derived belief following $d_{i}^{k}$ in the loop is an derived belief of sup $p(\bar{A})$, then the successive derived belief in the loop (note that as it is a loop, we are talking about all its derived beliefs) will also belong to sup $p(\bar{A})$ and, as sup $p(\bar{A})$ is invariable, our derived belief $d_{i}^{k}$ will also do so, thus contradicting our initial supposition.

So, if $d_{i}^{k} \in \sup p(\bar{L})$, we have $d_{i}^{k} \notin \sup p(\bar{A})$, in other words, both belief sets will be disjointed.

\section{REFLECTIONS}

1) Ideology is a fuzzy set of attitudes, beliefs, behavioral conventions, and basic assumptions and values that are shared by a group of people, and that influence each member's behavior and each member's interpretations of the 'meaning' of other people's behavior (Usó-Doménech.and Nescolarde-Selva, 2012; van Dijk, 2000). What a theory of ideology needs to explain, then, is precisely the dynamics that relate social members to ideologies and to the collectivities that are constituted by shared experiences, beliefs and ideologies. We need to know how individual membership, identification, allegiance, solidarity and active participation are being defined in relation to such a collectivity and its organization. It should be examined how groups may grow and decay as a result of the actions and participation of individuals. That ideological groups may be quite loose or fuzzy in their definition, and their membership or adherence flexibly defined in terms of the interplay between personal beliefs and socially shared beliefs, does not mean that we can dispense with the social dimension of ideologies in terms of groups. It is this interface between the individual and the group that is one of the theorical problems that need to be examined in a theory of ideologies.

Instead of defining belief systems and ideologies as the basis of all social cognition, we will now assume that general, cultural knowledge is the basis of all group-specific beliefs, including ideologies. Such cultural knowledge, or cultural common-ground, may be defined as the fuzzy set of those beliefs that are shared by (virtually) all competent members of a culture, and that are held to be true by those members by similarly shared criteria of truth. Neither cultural nor group knowledge is a well-defined 
concept. They are essentially fuzzy, in the sense that there is no effective procedure to establish for each culture or group what beliefs they collectively share (or indeed which are only shared by part of the group). Yet, the notions are far from arbitrary, and a quite reliable test (and there are others) is a presupposition in discourse. Cultural knowledge may be presupposed in all types of discourse by all competent (adult, sane, etc.) members, except of course in all didactic and pedagogical discourse that serves to teach such knowledge. Indeed, many groups may have ideological "deviants" or "'dissidents". If this is the case, the notion of group may at least sornetirnes be distinct from the set of its individual members. Maybe "groupness" only requires that most or many of the members share some property. However, such fuzzy criteria also make groups rather like fuzzy sets rather than strict sets of members.

One trivial answer already formulated is simply quantitative. That is, an ideological group can be defined as such if most of its members share most of the propositions that define the ideology, where the fuzzy quantifier "most" may be assigned any value between, say, seventy-five per cent and 100 per cent. Somewhat less trivial would be to replace the quantifier for the number of propositions by the set of substantive beliefs or "core" ideological propositions, namely, those that are the specific, defining or prototypical fundamental beliefs of a group. This is relatively straightforward of course for groups defined primarily by their ideologies.

2) In previous work (Nescolarde-Selva and Usó-Doménech, 2014, ) developed the theory that belief systems have a topological structure. Nevertheless, from where do these mathematical structures come? In the case of structures of visual materialization the answer seems clear. However, it is not so in the world of beliefs and ideas. Unless we accept the philosophy of Plato, freeing the world of ideas from the human being, beliefs, both substantive and derived beliefs have a material origin, inserted in the human brain. This can be considered from two aspects: psychological and linguistic:

1) Associated with the topological points is a family of open sets that cover space, like the response fields that constitute neighbourhoods of the actual neurons in the brain. The key point is that there are certain invariants associated with a topology that remain unchanged under the transformations. In the case of the visual field, the transformations are the distortions imposed by viewing conditions. The objects in the visual field are recognized as what they are in their own right no matter how their appearance may be distorted by viewing conditions: near or far, right-left, up-or-down in the field of view, rotated, moving, or viewed obliquely or binocularly. In addition, a tune is still recognizable even if it is shifted in key or changed in loudness, or heard binaurally. This invariance constitutes the psychological constancies. Lacking constancy invariance, you would always be moving through a surrealistic world of perpetually deforming, rubbery objects. For the visual system, it is axiomatic that an object is determined by its bounding contours, and it is the invariance of these under different viewing conditions that determines constancy and form memory (Lewin, 1936). This brings us to the blessed domain of Lie transformation groups, denoted symbolically by the mapping $G X T \rightarrow G$, where

$\mathrm{G}$ is a mathematical group and $\mathrm{T}$ is a manifold (Text). $\mathrm{G}$ is also continuous and is a manifold just like space-time. Now think of a visual contour as a path-curve generated by the transformation group action, and choose some point on it. Call this the identity element of the group. Draw a tiny tangent line to the curve at that point. This is the infinitesimal transformation of the continuous or Lie 
group. The infinitesimal transformation is embodied in a Lie derivative, which "drags the flow along the path-curve", the so-called "orbit"--in this case the visual contour of $\mathrm{T}$. If $£$ denotes the Lie derivative and $\mathrm{f}$, the visual contour, then invariance of the contour under the transformation group is shown by its being annulled by the action of the Lie derivative: $£ f=0$, or by its being handed on as a "contact element" for further processing: $£ f=g(f)$. These operations characterize psychological constancy.

2) In addition, neurological processes are organized and sequenced through language; hence, language reflects the way each person perceives the world. Being a psycho-biological process, one could say that mental maps are a sort of biological path along which words travel. The mental representations of individuals depend on their experiences, culture, ideology and physiology, among other things. Language refers to the way individuals make use of verbal expression to communicate experience, and this is done with the structure implicit in their own language.

Each of the substantive beliefs are propositions and these are formed by concepts. A concept is an analytical definition, an abstraction formed in the mind of a subject belonging to a particular semiotic system $S$. The different schools of philosophy have different contradictory views on the epistemological meaning of the concept. Here, the concept is the main component of rational thought in the attempt to apprehend reality. The concept is the union of denotative and connotative significances (Usó-Doménech and, 2012; Nescolarde-Selva and Usó-Doménech, 2014 ${ }^{\mathrm{a}, \mathrm{b}}$ ). And the connotative significance of a concept is different according to the World vision of any particular belief system. And not only that, but within a World vision, each individual may have a different connotation of the concept itself.

In this paper it is suggested by the authors that this structure is also fuzzy. The idea of building a fuzzy topology (Liu, andLuo, 1997) of belief systems and ideologies can be an exciting task.

\section{REFERENCES}

Alchourrón , C., P. Gärdenfors , and D. Makinson, 1985. On the logic of theory change: Partial meet contraction and revision functions. Journal of Symbolic Logic 50: 510-530,.

Béziau, J.Y.1997. What is many-valued logic? Proceedings of the 27th International Symposium on Multiple-Valued Logic, IEEE Computer Society, Los Alamitos, pp. 117121.

Borhek, J.T.; Curtis, R.F. 1983. A Sociology of Belief; Robert E. Krieger Publishing Company: Malabar. Florida.

Cignoli, R. L. O., D'Ottaviano, I, M. L. and Mundici, D., 2000. Algebraic Foundations of Many-valued Reasoning. Kluwer.

Eliade, M. 1978. A history of Religious Ideas. Volume I: From the Stone Age to the Eleusinian Mysteries. University of Chicago Press: Chicago. 
Esteve, P.F., and Lloret, M. 2006 - 'Coverage and invariability by structural functions,' International Journal of General systems, 35, 699-706.

Esteve, P.F., and Lloret, M. 2006 ${ }^{\mathrm{b}}$. 'Coverage, Invariability and orbits by structural functions Kybernetes, 35 (7-8), 1236-1240.

Gardenfors, P. 1988. Knowledge in Flux. MIT Press.

Lewin, K. 1936. Principles of Topological Psychology. New York and London. McGraw-Hill.

Liu, Y.-M., Luo, M.-K. 1997. Fuzzy Topology. Advances in Fuzzy Systems Applications and Theory, vol. 9, World Scientific, Singapore.

Lloret-Climent, M., Nescolarde-Selva, J.A., and Pérez-Gonzaga, S. 2014. Coverage and invariability in fuzzy systems. International Journal of General Systems. 43. 96-104. Malinowski, G. 2001. Many-Valued Logics, in Goble, Lou. Ed., The Blackwell Guide to Philosophical Logic. Blackwell.

Miller, D, M. and Thornton, M. A. 2008. Multiple valued logic: concepts and representations. Synthesis lectures on digital circuits and systems 12. Morgan \& Claypool Publishers.

Nescolarde-Selva, J., Vives Maciá, F., Usó-Doménech, J.L., Berend, D. 2012 .An introduction to Alysidal Algebra I. Kybernetes 41(1/2), pp. 21-34.

Nescolarde-Selva, J., Vives Maciá, F. Usó-Doménech, J.L., Berend, D. 2012 ${ }^{\text {b.An }}$ introduction to Alysidal Algebra II. Kybernetes. 41(5/6), pp. 780-793.

Nescolarde-Selva, J., Usó-Doménech. J. L. 2012. An introduction to Alysidal Algebra III. Kybernetes. 41(10), pp. 1638-1649.

Nescolarde-Selva, J. A and Usó-Doménech, J.L. 2013ㄹ. Topological Structures of Complex Beliefs Systems. Complexity. DOI: 10.1002/cplx.21455. Vol 19, 1. 46-62.

Nescolarde-Selva, J. and Usó-Doménech, J. 2013르. Topological Structures of Complex Belief Systems (II): Textual materialization. Complexity. 19 (2). pp. 50-62. DOI: 10.1002/cplx.21476.

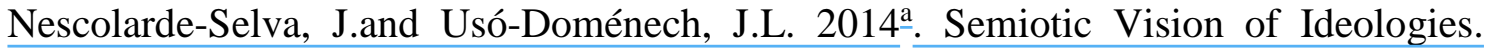
Found Sci, 2013; DOI:10.1007/s10699-013-9329-9. Vol 19. 263-282.

Nescolarde-Selva, J.and Usó-Doménech, J.L. 2014 . Reality, system and impure systems. Found Sci; DOI:10.1007/s10699-013-9337-8. Vol 19, 289-306.

Usó-Doménech, J.L.and Nescolarde-Selva, J. 2012. Mathematic and Semiotic Theory of Ideological Systems; Editorial LAP. Saarbrucken: Germany. 
Usó-Doménech, J.L. and Nescolarde-Selva, J. and Pérez-Gonzaga, S. 2014. Truth values in t-norm based systems many-valued fuzzy logic. American Journal of Systems and Software.

van Dijk, T.A. 2000. Ideology: a multidisciplinary approach. SAGE Publications Ltd. London.

Zadeh L.A. 1965. Fuzzy sets. Inform Control. 8:338-353. 